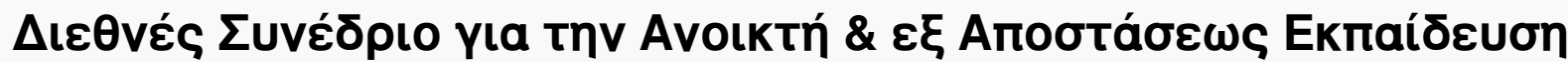

Tóp. 5, Ap. 2A (2009)

Open and Distance Education for Global Collaboration \& Educational Development

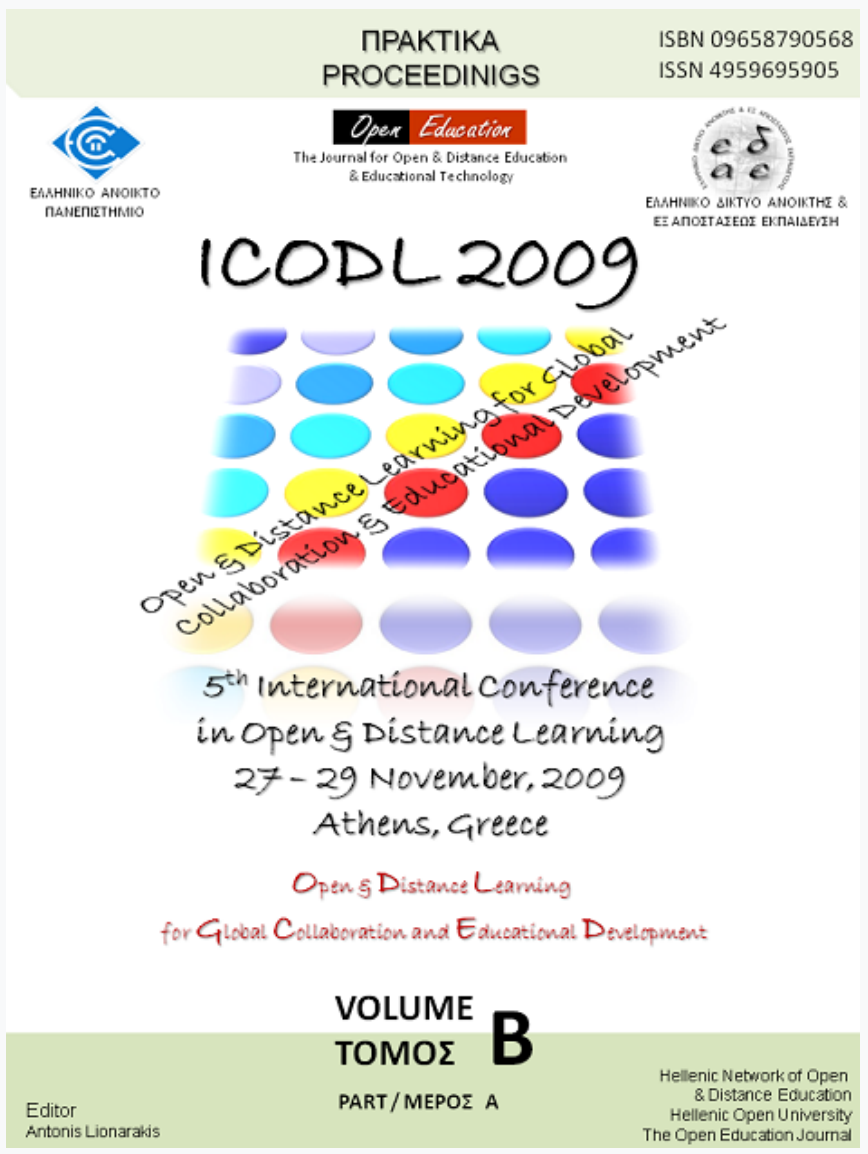

Technologically supported E-learning Systems in Higher Education

Victoria MYRONI, Andreas POMBORTSIS

doi: $10.12681 /$ icodl. 445 


\title{
Technologically supported E-learning Systems in Higher Education
}

\section{Victoria MYRONI}

Department of Computer Science

Aristotle University of Thessaloniki,

54124 Thessaloniki, Greece.

myroni@csd.auth.gr

\section{Andreas POMBORTSIS}

\author{
Department of Computer Science \\ Aristotle University of Thessaloniki, \\ 54124 Thessaloniki, Greece. \\ apombo@csd.auth.gr
}

\begin{abstract}
European higher education institutions have realized that today's society requires elearning systems that are flexible, have lifelong educational accessibility and which are cost effective. Since Europe cannot stand graduates' high unemployment rates, higher education institutions have to equip people with the skills for life long learning offering them a greater choice of learning paths. The Knowledge commercialization generates opportunities for new markets and poses a threat for new competitors to universities.

We focus on integrating a set of interoperable network services based on a Service Oriented Architecture (SOA) approach according to appropriate standards to succeed a flexible and powerful content management.

A platform is proposed to support the interoperability between the proposed and delivery platforms according to the specification of the services. Towards an open system the necessary components are interfaces to content repositories, reuse of existing metadata descriptors and a flexible data model for learning resource descriptors. The main objective of this platform is to analyze, search and present information retrieved from the web. It also touches on some important developments, such as Open Educational Resource movement, open research archives and global elibraries such as Europeana. Digital Libraries as information economies have a major influence on the design of future e-learning systems.
\end{abstract}

\section{Introduction}

Looking at existing e-Learning platforms the major problem is that most of them impose dependencies on content and system functionality which are either system component dependencies or data format dependencies.

E-learning platforms integrate functions such as authoring, course, access, user management, content retrieval, presentation and configuration. These functional components usually are tightly coupled resulting in integrating a new personal learning environment or publication subsystem to be really hard. The absence of integration activities between open source e-Learning platforms which are similar in terms of functionalities and implementation technologies is critical since most of the open source projects possess human resources.

In addition many e-learning systems organize and store the course structures according to the specific functionality of the system, having courses not to be platform independent. Packaging standards such as LTSC and SCORM exist on learning objects. Open Source community offers numerous e-Learning platforms of 
both types: Learning Management Systems (LMS) and Learning Content Systems (LCS). General purpose OS intermediaries such as SourceForge, ObjectWeb or CampusSource reduce the cost to locate such e-Learning platforms. Still, it is impossible to directly compare the functionalities of those OS software products without performing detailed testing on each product.

The remainder of this paper is organized as follows. Section 2 refers to the interoperability between e-learning platforms. Section 3 outlines the integration aspects into e-universities modules. Section 4 emphasizes on the work that needs to be done on functional requirements based on SOA systems. Section 5 focuses on open educational resources: Challenges-Examples. In Section 6 library services- quality of Service and economic issues are described. Section 7 concludes the paper.

\section{Interoperability between e-learning platforms}

Today's e-Learning Programmes explore the possibility of developing a more flexible service oriented approach to technical infrastructures for e-learning. Metadata can be saved into library records expanding the role of a library as a research tool rather than a mere locating device (Grant Campbell and Fast, 2004).

In the past many organizations like Microsoft tried to make specifications and tools for Application to Application interactivity, like the COM and CORBA. These initiatives were in the general concept of Service Oriented Architectures (SOA) where functionality is organized in discrete packages. Each package delivers functionality that targets specific problem (UNIVERSAL, 2002).

Recently the open standards for SOA made Sun, IBM and Microsoft add open standards to their approaches to SOA in the form of XML and SOAP. The strength behind XML is that its data is self describing. On the other hand the Simple Objects Application Protocol (SOAP) is a communication protocol that defines how the self describing XML data is transmitted from one point to another.

In this context the basis of the proposed platform for Interoperability with various elearning platforms in an A2A fashion is depicted in the figure 1. Using Web Services as an integration technology would be inadequate, since Web Services can only be used to make different type of implementation technologies interoperable. In this area Web Services help platforms to become interoperable with others in order to support Service Oriented Architecture (SOA). They can use services offered by other platforms easily. Each platform can use functionalities and services offered by other platforms straightforwardly, regardless of different implementation technologies used by each platform. For example Fig. 1 shows how interoperability between four different open source Learning Management Systems is established through web services. However it is almost unfeasible for an institution to install, manage and maintain four different LMS, implemented with different technologies to cover the needed e-Learning infrastructure. In many cases the institution chooses one LMS, depending on the implementation knowledge of its human resources and extends it in case of adding new functionalities by its internal personnel.

The proposed interface's platform (Ganchev, 2002) in order to maintain its role as a mediator service between the various e-learning content servers and services needs an implementation of an integration system open and generic for a wide number of environments. The suggested design allows the development of a collection of functions bundled together to deliver specific functionality to web based applications. This set of functions is platform's Web Services and will be developed within the 
framework of the Web Services Technology in order to be compliant and interactive with any other web services built which are based on open standards.

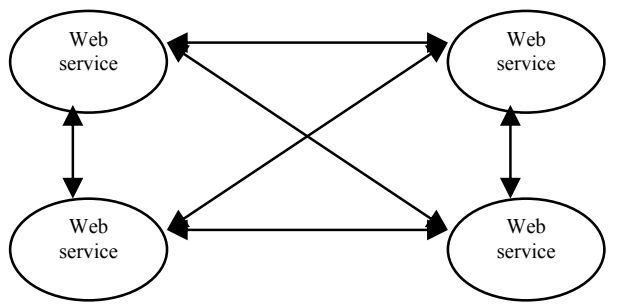

Figure 1. Integration by using web-services

With platform's Web services all the basic data of various e-learning platforms can be passed automatically to the proposed platform in a XML document driven by a SOAP client.

The programming language for the platform is Java which offers class libraries for XML and SOAP support. The web server supports JavaServer Pages and Servlets and the specific Java class libraries in order to be accessible from the proposed platform. It is very critical to define what functions the platform's Web Services will perform, which data will exchanged and when and under which procedures (EDUXCHANGE, 2005) . In addition to this the business logic must be very clear and must be included in Web Service Description Language (WSDL) documents i.e. XML documents that describe the type of web service, its endpoint URL which identifies the location of the remote service' s client connection point, methods that identify the remote web service, input parameter information and return types. These WSDL documents will be saved by the server on the Universal Dynamic Discover Integration (UDDI) registry that the application runs and will be accessible from the web interface of the proposed platform, giving information to the users of this platform on how they can achieve interoperability of their application with this platform. The Dynamic Discover Integration directory is a directory for Web Services that announces them to all the users for changes, once published.

For the connection with a remote web service it is necessary to know about its endpoint and namespace. Information about implementation details, such as which programming language or which host server is used is not needed. A SOAP Java class will create an XML document Request document filled with parameters and method details. This XML request will be addressed to the remote SOAP client . The remote SOAP Client will activate an object which is waiting for XML Requests and will invoke a method and assign the results to an XML Response document going back to the platform's SOAP client. Then a parameter object could start collecting data from platform's databases and sending them to the remote application in XML format using the http protocol.

\section{Integration aspects into e-universities modules.}

Web services can be used to implement a Service Oriented Architecture (SOA). Their focus is to create functional blocks independent of platforms and programming languages accessible over Internet. These can play the role of a Service provider or Service Broker or Service requestor which can be used in the case of an e-University as follows: 
1. The e-university (Myroni and Pombortsis, 2007) as a service provider must decide which services to expose (i.e. resource discovery, resource management, authentication, authorization), how to exploit them for other values, to decide what category the service could be listed for a broker service and what type of agreement is required for these services to be used.

2. The service broker is responsible for making the Web service interface and implementation access information available to any potential service requestor (Myroni and Pombortsis, 2008). Applications built for an e-university must be used in a supply chain and be exposed to business partners (i.e. brokers) who might compose, combine, and encapsulate them into new applications. This is the notion of the service value-net. The Universal Description Discovery and Integration (UDDI) specification defines a way to publish and discover information about Web services. Other service broker technologies include for example ebXML (Electronic Business using eXtensible Markup Language) and those based on the ISO/IEC 11179 Metadata Registry (MDR) standard.

3. The service requestor or Web service client locates entries in the broker registry. The term Web service definition refers to clients and servers that communicate using XML messages according to the SOAP standard. There is also a machine readable description of the operations supported by the server (i.e an application or device that performs services for connected clients as part of a client-server architecture) written in the Web Services Description Language (WSDL). The latter is necessary for automated client-side code generation in many Java and .NET frameworks. Some industry organizations, such as the WS-I, use both SOAP and WSDL in their definition of a Web service.

Web service location, integration and mediation can become dynamic, with software agents (JISC, 2006) able to reason about the functionalities provided by different web services, to locate the best ones for solving a particular problem, to automatically compose the relevant web services to build applications dynamically (Dominique, 2004). In the above case as an example the user requests from a service (Integration Service), types of courses, their prices and their description, the providers' addresses and their services and finally the supply of only the necessary output. Integrating services into one service implies discovering services that provide one or more functions; retrieving the definition of these functions, integrating them into a final service and solving data type constraints of the concepts included in the definitions of the functions (Myroni et al, 2008).

The Semantic Web requires relational metadata, i.e., metadata that describe how resource descriptions instantiate class definitions and how they are semantically interlinked by properties. Several case studies have been carried through that build on this idea of the Semantic Web, in order to provide intelligent applications to make knowledge about researchers, about companies and markets, and about research papers accessible by semantic means.

\section{Functional Requirements based on SOA Systems}

The model proposed in this section is loosely based on the IMS Abstract Framework. However, while the IMS Abstract Framework is specifying methods to use currently available techniques to build course management and e-learning systems, the SENSORIA e-university case study (SENSORIA, 2005) builds service-oriented 
architectures with a strong formal and semantic foundation by serving as a testbed for new tools and techniques.

The following scenarios are still open research problems:

- $\quad$ Open-ended systems: Most of the current techniques for analysing, modelling and reasoning about service-oriented systems fit to situations where a lot of designtime information about services is available, e.g., the interfaces of all services are known, and there is a fixed number of different configurations specified at design time. Future systems should be able to work reliably in a larger number of situations, e.g., they should allow the integration of previously unknown services into an existing system, or be able to adapt their behaviour to new requirements

- Common semantic basis: Several different formalisms at different levels of abstraction are necessary to describe and reason about service-oriented systems, e.g., constraint systems.It should be possible to relate these different descriptions to a common semantic foundation based on the SENSORIA SOA ontology.

The SOA Working Group has been developing and fostering common understanding of SOA in order to facilitate alignment between the business and information technology communities. The SOA Ontologies define the concepts, terminology and semantics of SOA in both business and technical terms, in order to:

i) Create a foundation for further work in domain-specific areas,

ii) Enable communications between business and technical people,

iii)Enhance the understanding of SOA concepts in the business and technical communities

v) Provide a means to state problems and opportunities clearly and unambiguously

vi) Potentially contribute to model-driven SOA implementation, which will facilitate SOA adoption.

In this section we address some implementation issues for the architecture. The university and e-learning system is structured as a collection of dynamically orchestrated services. We present e-learning systems that can be used by an euniversity, which can dynamically change as new services become available. Therefore the functionality provided by the discovery mechanism is used to describe services is an essential factor for determining the adaptivity of the e-university system.

- Services check for the availability of services matching their requirements for other services only when they register themselves.

- The services vary in the sophistication they require from the discovery mechanism. While simple services may rely on discovering a service with a precisely determined interface description, other services may be goal-driven and use semantic information to discover a set of services which they can use to satisfy their requirements.

\section{Open Educational Resources: Challenges - Examples}

The initiative is known as the Open Content Initiative or as the Open Educational Resources (OER) Initiative. The most inclusive term is Open Educational Resources (OER). "Open content" does not mean necessarily educational. The description of Open Educational Resources (OER) from the Hewlett website define Open Educational Resources to be teaching, learning, and research resources that support access to knowledge and reside in the public domain and can be freely used or repurposing by others. It targets students, educators and self-learners everywhere. 
According to OER technology in general and the World Wide Web in particular provide an extraordinary opportunity for everyone to share, use, and reuse knowledge. OER are the parts of that knowledge that comprise the fundamental components of education and content.

The goal is dissemination of high-quality content, innovative approaches to remove barriers to the creation, use, re-use and sharing of high-quality content and projects that seek to improve understanding of the demand for openly available content.

There is an estimation that of the total of $\$ 68$ million in grants, $\$ 43$ million has gone to the creation and dissemination of open content and \$25 million into reducing barriers, understanding and stimulating use. Of the total, about $\$ 12$ million has gone to non-U.S. institutions primarily in Europe, Africa, and China for capacity building, translation, and/or stimulation of established institutions such as the Open University in the United Kingdom and Netherlands, so they will be more aggressive in providing open content. About half of the $\$ 12$ million has gone to enhance the ability of developing countries to take advantage of the open content and contribute to it (Atkins et al, 2007).

Most remarkable is the extent to which OER has moved to "educational institutions" to embrace a new culture of IT-enabled contribution and sharing. Now it goes well beyond the institutions. For example the OpenCourseWare Consortium reveals a collaboration of more than 120 higher education institutions and associated organizations from around the world "creating a body of open educational content using a shared model.

The OCW project at MIT provided high-quality open educational resources for free. It is a pioneering project that contributed to a growing diversity to both the topic and the level of available open courseware.

The Internet Archive is a nonprofit organization dedicated to building and maintaining a free and openly accessible online digital library, including an archive of the Web. With offices located in the Presidio in San Francisco, California, and data centers in San Francisco, Redwood City, and Mountain View, CA, the archive includes "snapshots of the web" (archived copies of pages, taken at various points in time), software, books, and other recordings. To ensure the stability and endurance of the Internet Archive, its collection is mirrored at the Bibliotheca Alexandrina in Egypt, so far the only library in the world with a mirror. The IA makes its collections available at no cost to researchers, historians, scholars, and the general public. It is a member of the American Library Association and is officially recognized by the State of California as a library.

Europeana (Euroreana, 2008) Europe's multimedia online library opened to the public on 20/11/2008. Internet users around the world can now access more than two million books, maps, recordings, photographs, archival documents, paintings and films from national libraries and cultural institutions of the EU's 27 Member States. Europeana opens up new ways to anyone interested in literature and will have free and fast access to Europe's greatest collections and masterpieces in a single virtual library through a web portal available in all EU languages. In 2010, Europeana will have interactive zones such as communities for special interests. Between 2009 and 2011, some $€ 2$ million per year of EU funding will be dedicated to this. The Commission also plans to involve the private sector in the further expansion of Europe's digital library. In September 2007, the European Parliament supported, in a resolution voted by an overwhelming majority, the creation of a European digital library. 


\section{Library Services -Quality of Service and Economic Issues}

In today's e-learning Systems consider information-agents who provide searching and presentation services and users who request for services. In this case, one can envision suppliers and information-agents competing to provide services for information storage, searching, access and presentation. In providing such services, several issues arise; among them are socio-economic and cultural aspects of pricing information objects and Quality of Service (QoS) to access and view these objects. These issues play an important role in allocating resources such as processing time, network bandwidth and $\mathrm{I} / \mathrm{O}$, which are distributed (and owned) among various players in the economy. In this case the interactions among the players, service models, pricing, charging mechanisms and corresponding implementation issues are taking place.

For example in designing service mechanisms for accessing information objects in digital libraries, several issues arise. One of them is the issue of pricing services (and multiple service levels) to access information objects, and accounting and billing users for QoS.

Library services to support adult and lifelong learning have developed in response to changes in the higher education system (distance learning, part-time study, franchising of courses), with developments in adult and further education and open learning, and in response to demands from independent learners. In further and higher education, evidence from surveys of non-traditional students indicates the importance of library and information services to such students in supporting formal courses of study, whatever their mode of delivery.

After a period of time, intelligent services may seek out the most appropriate courses based on the learner's profile, requirements and information available from the multitude of learning providers and brokers. Our research uses the description of the content using metadata and presents a platform for the delivery of learning content to distribute and categorize academic content and to provide content catalogues and specific educational content to the target groups. In order to develop a framework for pricing services and charging/billing users for access, several questions arise and some of them are a) about the architecture b) about the economic framework c) about the players in the economy and their interactions. d) about the practical issues in implementing pricing schemes, and charging frameworks in large distributed Digital Libraries. There are several accompanying system level issues for storage, access of objects and performance demands on the local operating systems and distributed system (Sairamesh et al, 1996). Current networks and systems have limited resources (such as link bandwidth). Therefore, allocation of resources, such as processing time and network bandwidth, can be crucial in providing efficient services. In the future, bandwidth and processing time will be more than sufficient, and information objects will be priced based on the demand for them rather than on the Digital Library system that houses them. We consider a Digital Library System to be a complex computer system that provides storage, indexing, and access services to various information objects. In this paper, we assume that a set of resource allocations make up a service. An information object could also be a resource, but we do not price information objects in our case.

\section{Conclusions}


The higher education has proven it can produce high quality and innovative software. However there is a sizable community of users who are dissatisfied with the cost, the performance and the control of currently available options. Open source and interoperable software is a viable solution to this problem. However developers working in the commercial world do not have a nuanced appreciation of the ways in which software is used in higher education. The relatively small size of higher education may lead to monopolization and in this case the role of SOA architectures is really important. Regarding to the openness we referred to the manner in which the educational software is produced and to its open characteristics. Our contribution is the design of a platform to support the area of Technology Enhanced Learning. The specialized knowledge of experts is captured, stored and transferred automatically to training course materials. Open content exchanges and open source collaborative content, are alternative offerings to the commercial offers of the traditional learning providers.

Web Services are part of the evolution of component development technology. The two key aspects of component systems are the ability to plug in required software functions from heterogeneous bits of code, and the ability to invoke these functions across platform, network, language, and other such boundaries. It has long been recognized that metadata is one of the most important aspects of components systems. There are three main issues related to student support. First, institutions should gather as much personal data as possible about individual students to make sure that the proposed educational material corresponds to their individual needs and capabilities. Second, institutions should provide the appropriate technological infrastructure to students to enable them to their study. Finally students should be provided with access to administrative information and technology support.

Among others the supportive role of a number of virtual learning systems to a new educational model through the e-universities is necessary. Institutions can benefit at three different levels, depending on the type of intervention (OPENGROUP, 2008):

- efficiency (existing processes carried out in a more cost-effective and timeeffective manner)

- enhancement (improving existing processes and the outcomes)

- Transformation ( positive change in existing processes or introducing new processes).

As educational organizations become more knowledge driven, learning and business process management can not continue to be treated as separate worlds (PROLEARN, 2005).

\section{References}

ARIADNE (2006). 'Ariadne Tools', Retrieved on 24 April, 2006 from http://www.ariadne.org

Atkins, D., Brown, J., Hammond A.(2007). "A Review of the Open Educational Resources (OER) Movement: Achievements, Challenges and New Opportunities"

Courant, P. and Griffiths R. (2006). "Software and Collaboration in Higher Education: A Study of Open Source Software“"

Grant Campbell, D., Fast, K. (2004). "Academic Libraries and the Semantic Web: What the Future May Hold for Research-Supporting Library Catalogues". The journal of Academic Librarianship, Vol. 30, Issue 5, pp 382-390

Dominique J., et al. (2004). IRS III: "A Platform and Infrastructure for Creating WSMO-based Semantic Web Services", in Proceedings of the Workshop on WSMO Implementations, 2004. 
Euroreana (2008). Retrieved on 20 June, 2009 from: http://ec.europa.eu/information_society/

EDUXCHANGE, (2005). European Commission Directorate General for Education and Culture, Retrieved on 13 May, 2005 from http://www.int-evry.fr/eduxchange/

Ganchev, I., O'Droma M., Mc Donnell F. (2002) "Component-based platform for a virtual university information system”, Draft Standard for Learning Object Metadata (LOM). Proposed Draft 6.4, Technical Report, IEEE LTSC.

IMS (2004). 'Instructional Management Systems Global Learning Consortium, Inc. IMS Web team', Retrieved on 11 November, 2004 from http://www.imsproject.org

IMS Digital Repositories Interoperability (2004), Core Functions Information Model Version 1.0 Final Specification

Myroni, V. and Pombortsis, A. (2007) "Higher-Level Learning Activities through Integration of Applications in E-Universities", in Lionarakis A. (eds) Forms of Democracy in Education: Open Access and Distance Education, Proceedings of ICODL $20074^{\circ}$ International Conference on Open and Distance Learning Volume A, pp 199-205.

Myroni, V. and Pombortsis, A. (2008) Networked Libraries Promoting Lifelong Learning, Proceedings of the $6^{\text {th }}$ International Conference on Networked Learning, pp 278-284.

Myroni, V., Pombortsis, A., Maglaris V. (2008) "Innovative Design Issues of a Distributed E-learning Environment", International Journal of Innovation and Regional Development, accepted for publication.

OPENGROUP (2008) Retrieved on 13 May, 2009 from at: http://www.opengroup.org/

PROLEARN (2005) Retrieved on 13 May, 2007 from: http://www.prolearn-project.org/

SENSORIA (2005) Retrieved on 22 November, 2008 from: http://www.sensoria-ist.eu/

Sairamesh, J. et al. (1996) Economic Framework for Pricing and Charging in Digital Libraries, D-lib Magazine, Feb 1996

UNIVERSAL (2002) Retrieved on 22 November, 2008 from:http://www.istuniversal.org

Wang M. and Hwang M. (2004) The e-Learning Library: only a warehouse of learning resources? The Electronic Library, 22(5), pp 408-415

JISC,(2006). 'Joint Information Systems Committee frameworks programme', Retrieved on 17 December, 2006 from http://www.jisc.ac.uk 